\title{
Cerebral amyloid angiopathy-related inflammation (CAARI): case report
}

\author{
Karol Adam Makarewicz ${ }^{1}$, Karolina Zaryczańska ${ }^{1}, K^{2}$ arolina Machowska-Sempruch ${ }^{1}$, Anna Bajer-Czajkowska ${ }^{1}$, \\ Piotr Gołofit ${ }^{2}$, Ewa Gabrysz-Trybek², Przemysław Nowacki ${ }^{1}$ \\ ${ }^{1}$ Department of Neurology, Pomeranian Medical University in Szczecin, Poland, ${ }^{2}$ Department of Radiology, Pomeranian Medical \\ University in Szczecin, Poland
}

\begin{abstract}
Cerebral amyloid angiopathy-related inflammation is a new disease entity whose proper diagnosis may be difficult due to the fact that the early phase and radiological image resemble other conditions such as intracerebral haemorrhage or proliferative disorder. Also, the brain biopsy, which is an important part of the evaluation to confirm the diagnosis and rule out mimics, cannot be performed in each patient. In this paper we present the case of a $58 y e a r o l d$ man who was correctly diagnosed with cerebral amyloid angiopathy-related inflammation (CAARI) based on the results of the histopathological examination of the brain tissue, which was possible mainly owing to the inclusion of the expansive process as the underlying cause in the initial differentiation. Further progression of the disease, despite applying treatment of increasing intensity in response to progressive deterioration of the patient's condition, revealed the multiplicity of clinical courses that a new and not easily pinpointed entity can take.

Key words: cerebral amyloid angiopathyrelated inflammation, cerebral amyloid angiopathy, $\beta$-amyloid.
\end{abstract}

\section{Introduction}

Cerebral amyloid angiopathy-related inflammation (CAARI), a relatively recently recognized disease entity, occurs in patients affected by cerebral amyloid angiopathy (CAA) [6,7], also referred to as congophilic amyloid angiopathy. CAA is a form of amyloidopathy in which $\beta$-amyloid deposits form in the walls of the small blood vessels located mainly in the subcortical region of the brain. The deposits are composed of products of the amyloid precursor protein cleavage. The process, controlled by $\beta$ - and $\gamma$-secretases, leads to the formation of $\beta$-amyloid and a few smaller peptides ( $A \beta-40$ and $A \beta-42$ ). Being laden with amyloid plaques aggregated on their walls the vessels become weakened and prone to rapture, which in turn leads to recurrent intracerebral haemorrhage. Familial (hereditary) and sporadic types of cerebral amyloid angiopathy have been distinguished to date. Its prevalence ranges between 30 and 40 cases per 100,000 people with most cases occurring in the $5^{\text {th }}$ or $6^{\text {th }}$ decade of life. It is considered to be the second most frequent cause of atraumatic intracerebral haemorrhage [6,7].

Apart from haemorrhagic foci typical of CAA, CAARI manifestations include inflammation areas and vasogenic oedema of the brain tissue with localized mass effect. Pathologic samples obtained through cortical biopsy in some patients revealed perivascular inflammation with the presence of multinucleated giant cells $[1,6]$. Other prominent clinical manifestations of CAARI are frequent headaches accompanied by epileptic seizures and a rapidly progressive dementia. 
The differential diagnosis of the disease includes primary CNS vasculitis, neurosarcoidosis, autoimmune encephalitis, and CNS malignancy [6,7]. Diagnostic criteria for CAARI [2] were first proposed in 2011. They were subsequently revised in 2014, and now are as follows [2,9]:

Probable CAARI (all of the below):

1. Acute or subacute onset of symptoms;

2. Age $\geq 40$ years;

3. Presence of $\geq$ of the following clinical features: headache, decrease in consciousness, behavioural change or focal neurological signs and seizures;

4. Magnetic resonance imaging (MRI) with patchy or confluent T2-weighted or fluid-attenuated inversion recovery (FLAIR) lesions, which are a) usually asymmetric, b) with or without mass effect, and c) with or without leptomeningeal or parenchymal enhancement;

5. Earlier CAA features: a) multiple cortical or subcortical haemorrhages or microhaemorrhages and/or b) recent or past lobar intracerebral haemorrhage;

6. The absence of neoplastic, infectious, or other causes. Definite CAARI (all of the above criteria met plus histopathological examination confirming the diagnosis):

1. Intramural or perivascular inflammation;

2. Amyloid deposits in the cortical vessels of the affected region.

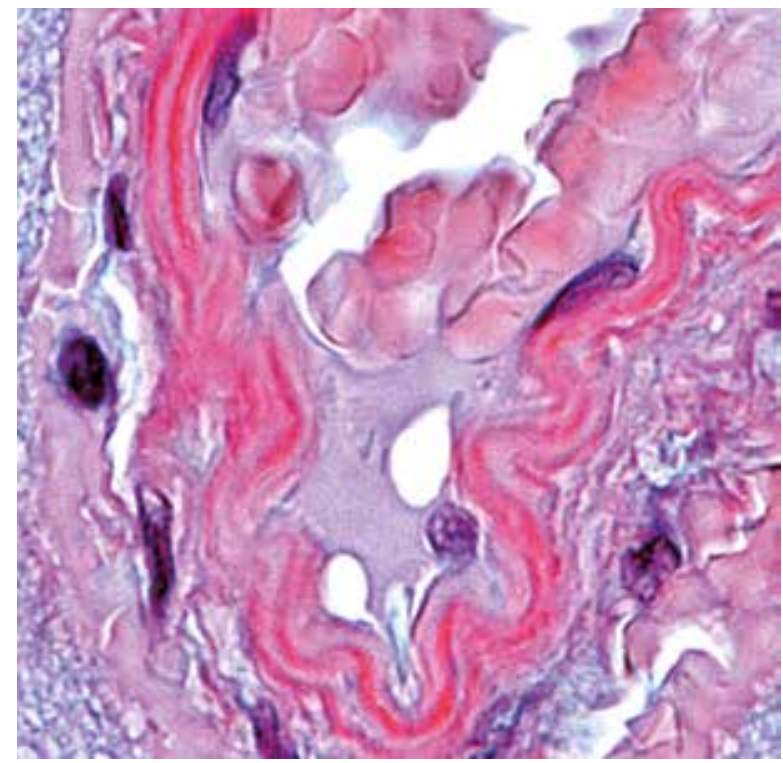

Fig. 1. Homogeneous intravascular eosinophilic deposits within the cortical arteriole. Congo red (400× magnification).
The aetiology of the disease is believed to be associated with autoimmune response. Treatment is based on immunosuppressive drugs with the majority of patients treated with corticosteroids, methotrexate, mycophenolate, mofetil, cyclophosphamide and intravenous immunoglobulin. Sources report a positive response to treatment and clinical improvement in approximately $50 \%$ of the patients whereas in around $33 \%$ of the diagnosed, the disease progresses inevitably leading to death. Attempts have also been made to try anti-amyloid drug treatment [4].

\section{Case presentation}

The subject is a 58-year-old unmarried and childless man with a basic vocational training in agriculture and no history of chronic diseases. First admitted to a neurological department in June 2017 due to the right-side focal seizures, which are secondary generalized, corresponding to pathological changes located within the left parietal lobe, visualized on computed tomography (CT) scans. CT scans revealed the presence of a hyperdense, non-enhancing focus in the left parietal lobe, suggesting a malignant change. Further diagnostic investigations using SPECT-MRI made us suspect low-grade glioma. Carbamazepine-related drug treatment was administered and the patient was referred for a diagnostic brain biopsy.

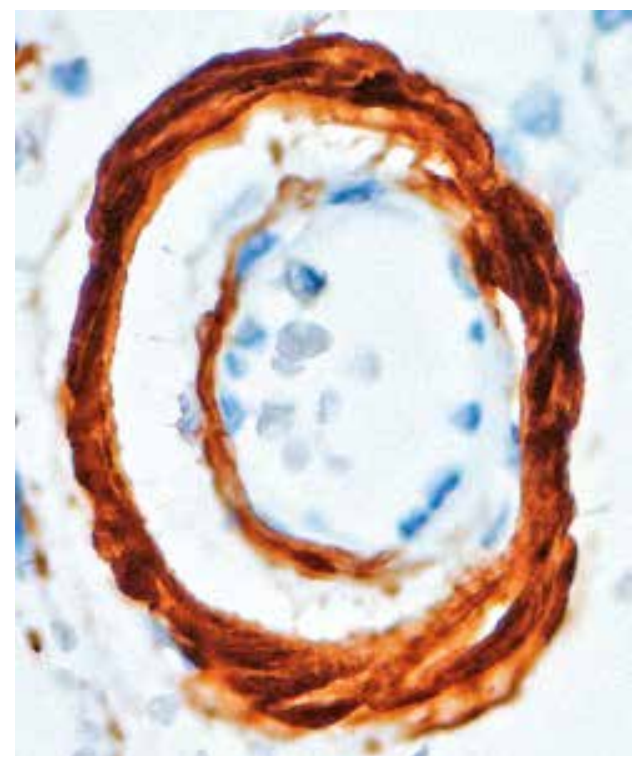

Fig. 2. $\beta$-amyloid deposits within the vascular wall and a barrel-like structure in the lumen of the maternal vessel (200× magnification). 


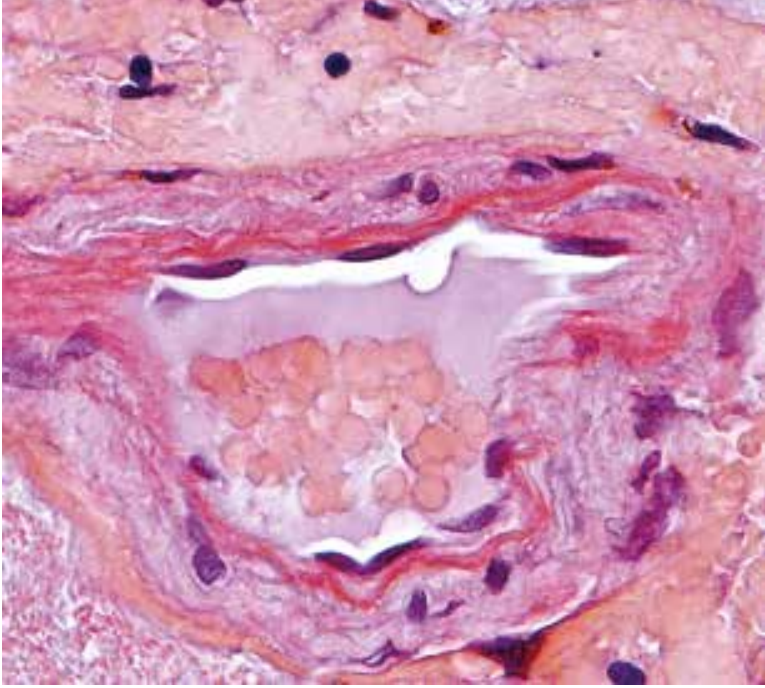

Fig. 3. Perivascular haemorrhage confined to the perivascular space. The vessel wall filed with congo-positive deposits. Congo red (200× magnification).

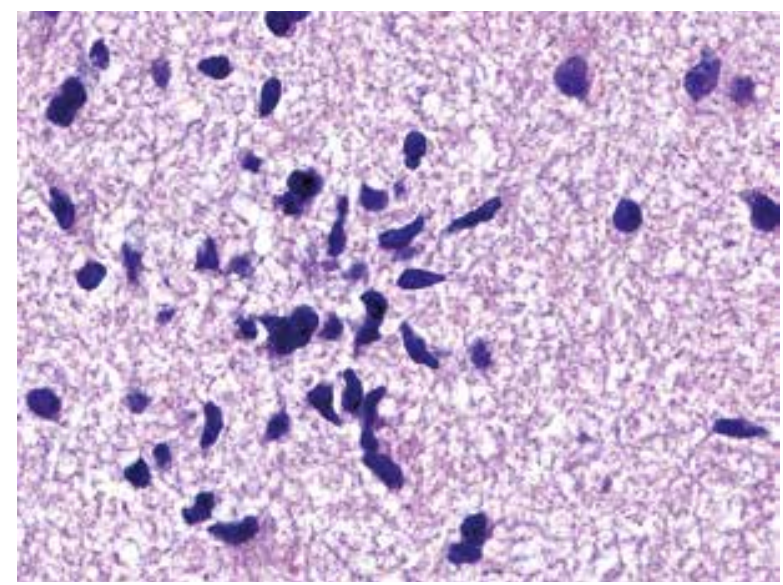

Fig. 5. Microglial nodule. Hematoxylin-eosin (400× magnification).

Examination of a small sample of biopsy material demonstrated the existence of homogeneous eosinophilic deposits located within the vessel walls in single meningeal and cortical arterioles (small sample size), Congo red-positive (Fig. 1), indicative of $\beta$-amyloid fibrils. In the lumen of some vessels, barrel-like structures were observed (Fig. 2). A number of perivascular haemorrhages confined to the perivascular space (Fig. 3) or penetrating into the adjacent brain tissue were also found. The image also showed the presence of perivascular T-lymphocyte infiltrations (Fig. 4), microglial nodules (Fig. 5) and intraparenchymal astrocyte-microglia reactivity (Fig. 6).

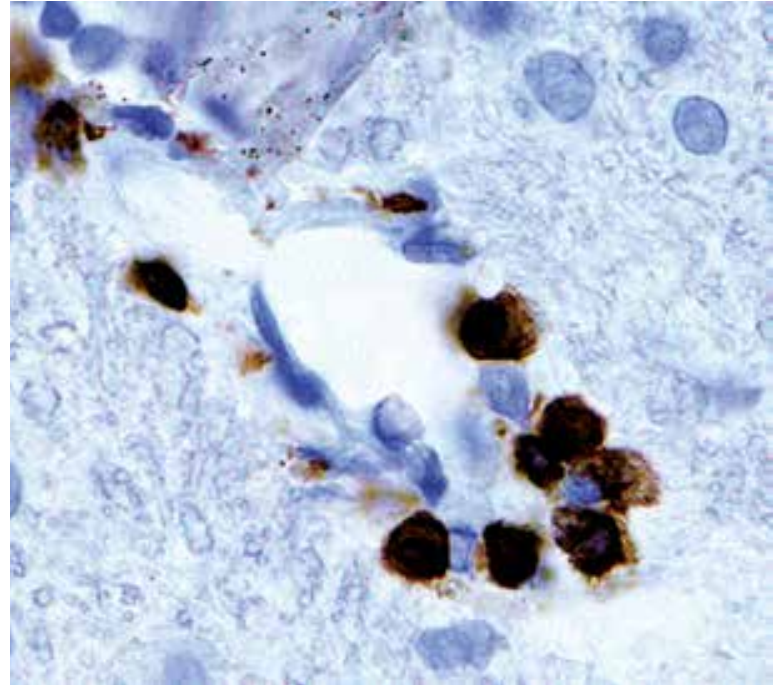

Fig. 4. Perivascular TCD3-lymphocyte infiltration (400× magnification).

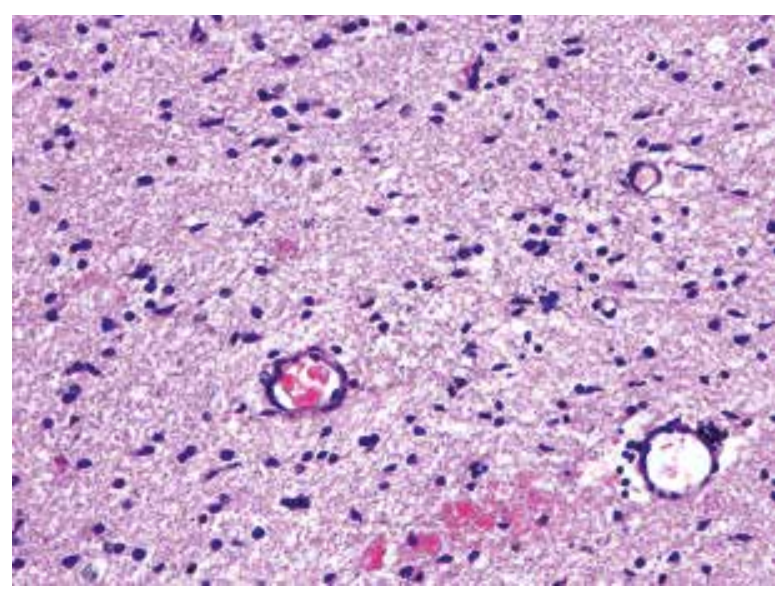

Fig. 6. Intraparenchymal astrocyte-microglia reactivity. Hematoxylin-eosin (200× magnification).

While in hospital the patient was diagnosed with hypertension and diabetes type 2. No symptoms of neurological deficits were recorded at discharge.

In June/July 2017, the patient underwent treatment in the Koszalin Hospital, Department of Neurology for right-sided hemiparesis. Diagnostic neuroimaging showed abnormalities resembling brain haemorrhage or tumour - the MRI scan revealed haemorrhage within the left parietal lobe in the place of the changes described previously, which corresponded to rightsided paresis. Tests carried out for onconeural antibodies (against $\mathrm{Hu}, \mathrm{Yo}, \mathrm{Ri}$, amphiphysin, Ma2, PNM2/Ta, anty-SOX1, Titin, Rec and CV2.1) and rheumatoid anti- 


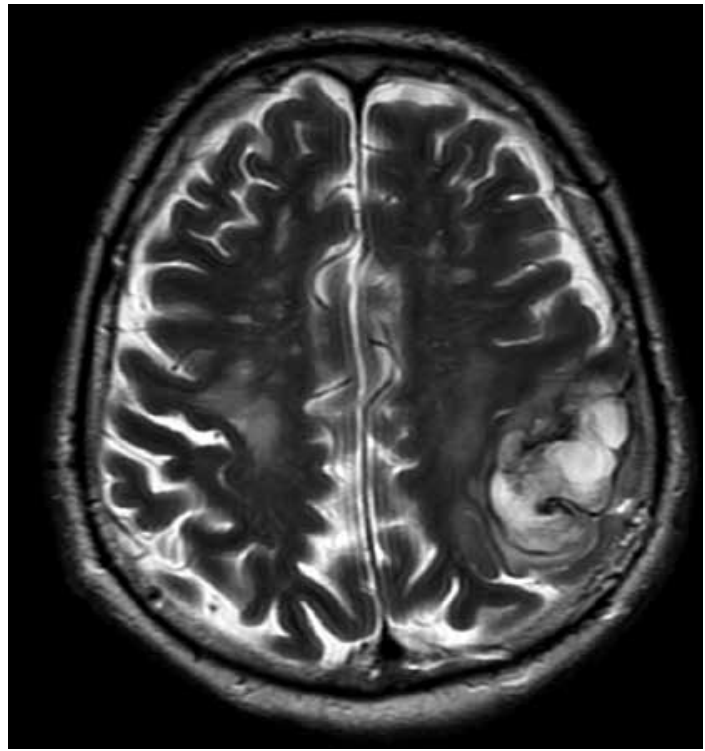

Fig. 7. Large lesion in the left fronto-parietal area with central and peripheral haemorrhages. Numerous hyperintense areas visible on both hemispheres.

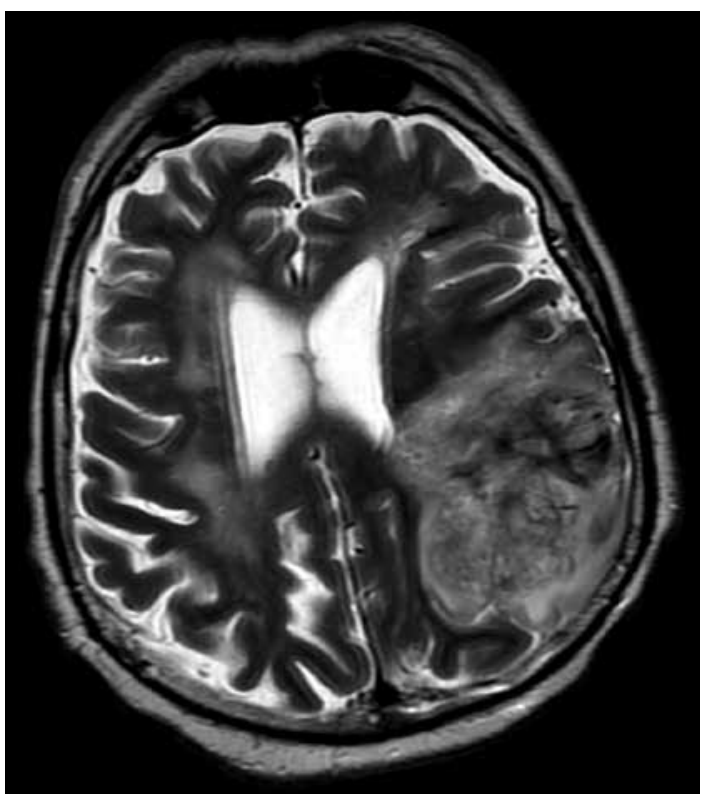

Fig. 9. MRI T2W scan taken one year later showing a new haemorrhage and small ischemic and haemorrhagic lesions.

bodies (against $\beta 2$ glicoprotein, proteinase 3, myeloperoxidase, anticardiolipin antibodies) yielded negative results and the man was discharged.

Soon afterwards, he was admitted to our clinic for the verification of the diagnosis. At admission he was

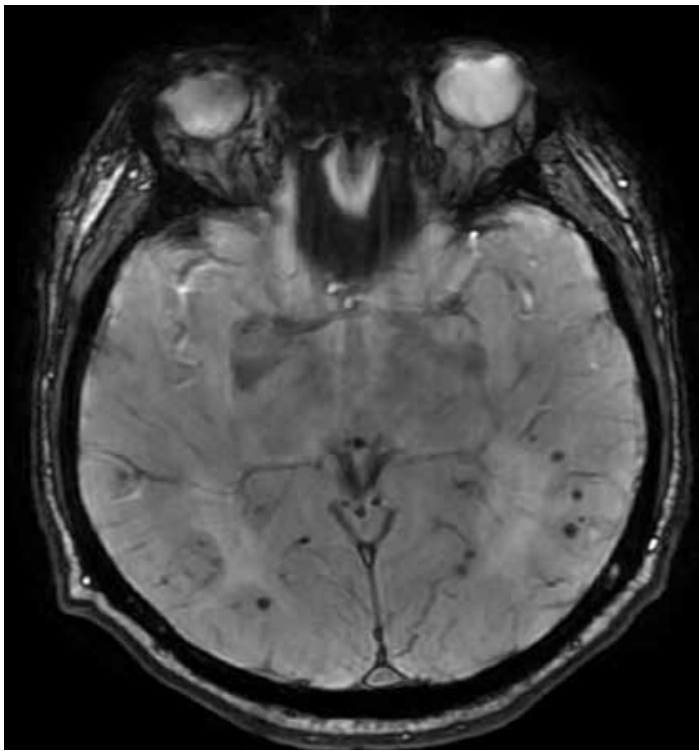

Fig. 8. SWI image of dispersed small foci of accumulated hemosiderin.

conscious, logical, oriented, cardiovascularly and respiratorily stable, blood pressure of $120 / 70 \mathrm{mmHg}$ and regular pulse of 72 beats per min. During his hospital stay, we detected residual motor aphasia and discrete right-sided hemiparesis. $\mathrm{C}$ reactive protein (CRP) was $18.85 \mathrm{mg} / \mathrm{l}$ [norm 0.00-5.00], alanine aminotransferase (ALT) - $54 \mathrm{U} / \mathrm{I}$ [norm 0-41], alkaline phosphatase (ALP) - 90 U/I [norm: 40-130], gamma-glutamyl transpeptidase (GGTP) - 453 U/I [norm: 9-60].

Further brain MRI studies revealed:

1. An area of inhomogeneous signal in T1 and T2-weighted images, dimensions $55 \times 34 \times 28 \mathrm{~mm}$, located in the left fronto-parietal region - a lesion with features of the central chronic and late subacute peripheral haemorrhage (Fig. 7);

2. Numerous confluent T2/FLAIR hyperintense areas in the white matter of both hemispheres (Fig. 7);

3. Pronounced, on SWI image, multiple foci of susceptibility artifact in both hemispheres suggesting presence of microhemorrhages (Fig. 8).

A cerebrospinal fluid test did not reveal any abnormalities. Ambiguity of the clinical picture called for yet another series of blood tests for onconeural and antineuronal antibodies (AntiAmpa GluR1GluR2, AntiGABA B, AntiNMDA, AntiDPPX, AntiCASPR2, Antilgl1). They yielded negative results.

Having analysed the entire clinical picture, especially the results of the biopsy clearly demonstrating 
an inflammatory component, we diagnosed the man with cerebral amyloid angiopathy-related inflammation (CAARI). Prednisone in a dose of $40-20-0 \mathrm{mg}$ was introduced. Due to the elevated levels of liver enzymes, anti-epileptic carbamazepine was replaced with levetiracetam.

In July 2017, the patient was again admitted to our clinic of neurology due to the deterioration of speech and general functioning. When interviewed his family confessed that the man had not been taking the prescribed medication regularly. Apart from previously observed psychomotor slowing, residual motor aphasia and discrete right-sided hemiparesis, the patient now demonstrated conspicuous dysarthria. No impairment in swallowing was found. Another MRI brain scan revealed a considerable regression of the previously described haemorrhagic focus in the left fronto-parietal area with the presence of hemosiderin deposits. The area of inhomogeneous signal in T1 and T2-weighted images, dimensions $51 \times 35 \times 28 \mathrm{~mm}$, and signs of chronic peripheral haemorrhage were still visible. An electroencephalography (EEG) test indicated the existence of discrete scattered lesions. During his hospital stay the patient was administered intravenous methylprednisolone, prednisone in a modified dose of 30-20-0 mg. A once-per-week 10-0-0 mg dose of methotrexate was also introduced. This was supplemented by the administration of motor and speech therapies. At discharge the man was self-sufficient regarding everyday activities, he demonstrated dysarthria and right-side supranuclear facial palsy.

It was not until October 2017 that the man was hospitalized again because of a progressive deterioration of speech and functioning. Prior to admission he experienced focal motor seizures of the right upper limb. He still was not taking the prescribed drugs on a regular basis. At admission he demonstrated psychomotor slowness, minor signs of motor aphasia, considerable dysarthria, severe right-sided paresis affecting the facial muscles and tongue, mild right-sided hemiparesis which was more conspicuous in the upper limb. The MRI scan did not differ significantly from that performed in July 2017. EEG showed paroxysmal changes in the left temporooccipital leads. Methotrexate and levetiracetam dosage was increased to $12.5-0-0 \mathrm{mg}$ once weekly and $2 \times 1000 \mathrm{mg}$, respectively.

In January 2018, the man stayed in hospital again due to further progressive deterioration of speech and functioning and more frequent episodes of focal motor seizures of the right upper limb. He was diagnosed with motor aphasia, considerable dysarthria, severe right-sided weakness of the facial muscles and tongue as well as markedly more severe rightsided hemiparesis necessitating the use of a crutch for walking. Like previously, the MRI test revealed the presence of an area of inhomogeneous signal on T1- and T2-weighted images, dimensions $55 \times 35 \times$ $28 \mathrm{~mm}$, located in the fronto-parietal region, within which a semi-circular subcortically located area of high signal intensity on T1-weighted image was described, indicative of a fresh haemorrhage (Fig. 9). A decision was made to gradually terminate the prednisone therapy and keep the dosage of the methotrexate unchanged. Dose of $10 \mathrm{mg}$ of escitalopram was introduced as the patient demonstrated concomitant depressive disorders. When leaving the hospital the patient walked with a crutch.

A month later, after reporting difficulty in swallowing and persisting focal motor seizures of the right upper limb, the man was admitted to hospital again. He still demonstrated motor aphasia, dysarthria, severe right-sided weakness of the facial muscles and tongue, but his right-sided hemiparesis was now replaced with right-sided hemiplegia. Another MRI examination was performed revealing in the left fronto-parietal region an irregular intracerebral haemorrhage surrounded by oedema. The patient's reluctance to take in food and the presence of concomitant pseudobulbar palsy necessitated the application of percutaneous endoscopic gastrostomy which was performed after obtaining relevant consent from the court. The ongoing treatment was modified by introducing mycophenolate mofetil $2 \times 1000 \mathrm{mg}$ and increasing the dosage of levetiracetam to $2 \times 1250 \mathrm{mg}$. Throughout his entire stay in the hospital the patient exhibited significant impairment of virtually all cognitive functions and was diagnosed with severe dementia. He was also unresponsive and failed to cooperate with the physiotherapist. The man was eventually discharged with the recommendation of family care and outpatient consultations regarding further treatment.

\section{Discussion}

CAARI is generally considered a condition that is too often misdiagnosed, which results from the fact that the dominant feature of the relevant imaging studies are heterochronous haemorrhagic and/or isch- 
emic foci, usually mistaken for CAA. Another difficulty regarding the identification and accurate diagnosis of CAARI is the fact that standard clinical investigations are rarely accompanied by confirmatory brain biopsy which is only performed when it is significantly reasonable to verify the diagnosis of suspected CAA. Histological examination of the brain tissue allows diagnosis based on an atypical inflammatory component which imaging studies often fail to visualize.

The only reason our patient was referred for the brain biopsy was a reasonable suspicion, based on the results of imaging tests, of the neoplastic process developing in the left parietal lobe. As the histopathological examination did not confirm this diagnosis, the patient was now believed to have developed an autoimmune inflammatory process concurrent with lesions of vascular origin, characteristic for CAA (presence of cerebrovascular $\beta$-amyloid depositions, perivascular haemorrhages and lymphocytic infiltration). $\beta$-amyloid plaques were not detected in the brain parenchyma. Their presence would be the evidence of frequently observed coexistence of the two conditions (Alzheimer's disease and amyloid angiopathy) where $\beta$-amyloid depositions play the principal role [8].

The differential diagnosis included primary angiitis of the central nervous system (PACNS) and amyloid $\beta$-related angiitis (ABRA). The hallmark of the former condition includes disturbances of consciousness, occasional psychotic symptoms and less frequently observed focal signs. A characteristic feature of ABRA is inhomogeneous contrast enhancement of the soft meninges resulting from the fact that they are the main site of lesion. Unlike CAA, though, intracerebral haemorrhages are less likely to occur $[10,11]$. Also, ABRA inflammatory infiltrations affect the wall of intracranial vessels and by this, together with $\beta$-amyloid depositions, contribute to its destruction. Hence the entity is referred to as angio-destructive inflammatory mediated disease. In CAA, the inflammatory infiltrations are of perivascular nature, do not directly inflict damage to the vessel walls which are covered by $\beta$-amyloid depositions (non-destructive angiitis) $[3,5]$. Even though the amount of the tissue sampled for histopathological examination was small, the analysis of the clinical picture, including the imaging studies, and the features of neuropathological lesions allowed us to diagnose CAARI. In both CAARI and ABRA, the treatment is based on immunosuppressive therapy [5]. The course of the disease varies. Apart from patients demonstrating regression or stabilization of symptoms, the literature describes those who have relapsed upon treatment termination. Mortality rates vary from a few to over ten per cent. Sadly, despite continuous and increasingly aggressive treatment our patient's condition kept deteriorating till his last hospital stay. His ultimate fate at the moment of preparing this publication remains unknown, but considering the progression of the disease, it can be assumed that the prognosis is rather poor.

\section{Disclosure}

The authors report no conflict of interest.

\section{References}

1. Auriel E, Charidimou A, Gurot ME, Ni J, Van Etten ES, MartinezRamirez S, Boulouis G, Piazza F, DiFrancesco JC, Frosch MP, Pontes-Neto OV, Shoamanesh A, Reijmer Y, Vashkevich A, Ayres AM, Schwab KM, Viswanathan A, Greenberg SM. Validation of clinicoradiological criteria for the diagnosis of cerebral amyloid angiopathy-related inflammation. JAMA Neurol 2016; 73: 197-202.

2. Chung KK, Anderson NE, Hutchinson D, Synek B, Barber PA. Cerebral amyloid angiopathy related inflammation: three case reports and a review. J Neurol Neurosurg Psych 2011; 82: 20-26.

3. Eng JA, Frosch MP, Choi K, Rebeck GW, Greenberg SM. Clinical manifestations of cerebral amyloid angiopathy-related inflammation. Ann Neurol 2004; 55: 250-256.

4. Hirohata M, Ono K, Yamada M. Non-steroidal anti-inflammatory drugs as anti-amyloidogenic compounds. Curr Pharmaceut Design 2008; 14: 3280-3294.

5. Kinnecom C, Lev MH, Wendell L, Smith EE, Rosand J, Frosch MP, Greenberg SM. Course of cerebral amyloid angiopathy-related inflammation. Neurology 2007; 68: 1411-1416.

6. Kirshner HS, Bradshaw M. The inflammatory form of cerebral amyloid angiopathy or "cerebral amyloid angiopathy-related inflammation” (CAARI). Curr Neurol Neurosci Rep 2015; 15: 54.

7. Mendel TA. Sporadyczna mózgowa angiopatia amyloidowa - patofizjologia, objawy, diagnostyka i leczenie. Pol Przegl Neurol 2015; 11: 163-172.

8. Nouth A, Borys E, Gierut A, Biller J. Amyloid-beta related angiitis of the central nervous system: case report and topic review. Front Neurol 2014; 5: 13.

9. Rajczewska-Oleszkiewicz C, Cyganek A, Stadnik A, Dziewulska D. Cerebral amyloid angiopathy-related inflammation - a case report presenting diagnostic difficulties. Neurol Neurochir Pol 2018; 52: 298-305.

10. Salvarani C, Hunder GG, Morris JM, Brown RD, Christianson T, Giannini C. A $\beta$-related angiitis comparison with CAA without inflammation and primary vasculitis. Neurology 2013; 81: 1596-1603.

11. Scolding NJ, Joseph F, Kirby PA, Mazanti I, Gray F, Mikol J, Ellison D, Hilton DA, Williams TL, MacKenzie JM, Xuereb JH, Love S. A $\beta$-related angiitis: primary angiitis of the central nervous system associated with cerebral amyloid angiopathy. Brain 2005; 128: 500-515. 